\title{
Reachable Set Estimation for a Class of Nonlinear Time-Varying Systems
}

\author{
Yuangong Sun ${ }^{1,2}$ and Fanwei Meng ${ }^{3}$ \\ ${ }^{1}$ School of Mathematical Sciences, University of Jinan, Jinan, Shandong 250022, China \\ ${ }^{2}$ College of Mathematics and Systems Science, Shandong University of Science and Technology, Qingdao, Shandong 266590, China \\ ${ }^{3}$ School of Mathematical Sciences, Qufu Normal University, Qufu, Shandong 273165, China \\ Correspondence should be addressed to Yuangong Sun; sunyuangong@163.com
}

Received 11 April 2017; Accepted 12 June 2017; Published 12 July 2017

Academic Editor: Pietro De Lellis

Copyright (C) 2017 Yuangong Sun and Fanwei Meng. This is an open access article distributed under the Creative Commons Attribution License, which permits unrestricted use, distribution, and reproduction in any medium, provided the original work is properly cited.

\begin{abstract}
This paper studies the problem of reachable set estimation for a class of nonlinear time-varying systems with disturbances. New necessary and (or) sufficient conditions are derived for the existence of a ball such that all the solutions of the system converge asymptotically within it. Explicit estimation on the decay rate is also presented. The method used in this paper is motivated by that for positive systems, which is different from the conventional Lyapunov-Krasovskii functional method. A numerical example is given to illustrate the effectiveness of the obtained result.
\end{abstract}

\section{Introduction}

The problem of reachable set estimation (bounding) for linear systems has been addressed in [1-10], to name a few. In all of the aforementioned works, the involved system was mainly aimed at linear time invariant systems. Moreover, the Lyapunov-Krasovskii functional method was most commonly used, which is usually invalid to time-varying systems because they lead to either unsolvable matrix Riccati differential equations or indefinite linear matrix inequalities.

Recently, Hien and Trinh considered the problem of reachable set bounding for linear time-varying systems with delay and bounded disturbances for the first time in [11]. By using a new approach which does not involve the LyapunovKrasovskii functional method, an explicit delay-independent condition for state bounding of the system was given in terms of Metzler matrix. The reachable set bounding for a class of nonlinear perturbed time-delay systems was investigated in [12], where the involved nonlinear term satisfies linear growth condition. Recently, state bounding for homogeneous positive systems of degree one with time-varying delay and exogenous input was studied in [13]. In this paper, we will further study the reachable set estimation for a class of nonlinear time-varying systems without satisfying the linear growth condition or the homogeneous condition of degree one. For the particular case when the system is positive, we first establish a necessary and sufficient condition for reachable set bounding. Then, we extend the result to the more general case by using the comparison principle.

Positive systems, whose state trajectories remain nonnegative for all time provided that initial states are nonnegative, have received much attention in recent years (see the books $[14,15]$ and the references therein). When dealing with stability problems of positive systems, an approach independent of Lyapunov-Krasovskii functional was commonly used in [1621]. Inspired by this, the paper will apply such a method to the reachable set estimation for nonlinear time-varying systems with disturbances. Necessary and (or) sufficient conditions have been established such that all the solutions of the system converge asymptotically within a ball determined by the upper bound of disturbances. Moreover, the explicit decay rate is also presented.

The paper is organized as follows. In Section 2, we present the notation used through this paper as well as preliminaries for our results. Section 3 then focuses on deriving explicit conditions under which all the solutions of the system 
converge asymptotically within a ball. Section 4 provides an illustrative example to show the effectiveness of the obtained result. The paper is concluded in Section 5.

\section{Preliminaries}

Throughout this paper, the following notation will be used. Let $\mathbb{R}^{n}$ and $\mathbb{R}^{n \times n}$ denote the set of $n$-dimensional real vectors and the $n$-dimensional real Euclidean space, respectively. The matrix $A \in \mathbb{R}^{n \times n}$ is said to be Metzler if all its off-diagonal entries are nonnegative. For $x \in \mathbb{R}^{n}$, we denote by $x_{i}$ the $i$ th coordinate of $x$. For two vectors $x, y \in \mathbb{R}^{n}$, we write $x>y$ if $x_{i}>y_{i}, x \geq y$ if $x_{i} \geq y_{i}, x \prec y$ if $x_{i}<y_{i}$, and $x \preceq y$ if $x_{i} \leq y_{i}$, $i=1,2, \ldots, n$. Let $\mathbb{R}_{+}^{n}=\left\{x \in \mathbb{R}^{n}: x \geq 0\right\}$. For $x=\left(x_{i}\right) \in \mathbb{R}^{n}$, let $|x|=\left(\left|x_{i}\right|\right) \in \mathbb{R}_{+}^{n}$ and $\|x\|_{\infty}=\max _{i=1,2, \ldots, n}\left|x_{i}\right|$. Given an $n$-dimensional vector $v=\left(v_{i}\right)>0$, the weighted $\infty$-norm of the vector $x \in \mathbb{R}^{n}$ is defined by $\|x\|_{\infty}^{v}=\max _{i=1,2, \ldots, n}\left(\left|x_{i}\right| / v_{i}\right)$. For $\varepsilon>0$, denote a ball by $\mathscr{B}(\varepsilon)=\left\{x \in \mathbb{R}^{n} \mid\|x\|_{\infty} \leq \varepsilon\right\}$.

Consider the following continuous-time nonlinear timevarying system described by

$$
\dot{x}(t)=\mathbf{F}(t, x(t))+w(t), \quad t \geq 0,
$$

where $x(t) \in \mathbb{R}^{n}$ is the state vector, $w(t):[0, \infty) \rightarrow \mathbb{R}^{n}$ is the unknown disturbance input, and the vector field $\mathbf{F}(t, x)$ : $[0, \infty) \times \mathbb{R}^{n} \rightarrow \mathbb{R}^{n}$ is continuous and locally Lipschtiz with respect to $x$, which ensures the existence and uniqueness of solutions of system (1) [22]. We now begin with the following definitions.

Definition 1. System (1) is said to be positive if, for any initial condition $x(0) \in \mathbb{R}_{+}^{n}$, the corresponding state trajectory $x(t)$ remains nonnegative for all $t \geq 0$.

The following proposition gives a sufficient condition guaranteeing the positivity of system (1).

Proposition 2. System (1) is positive if

$$
\begin{aligned}
w(t) & \geq 0, \\
F_{i}(t, x) & \geq 0,
\end{aligned}
$$

$$
t \geq 0, i=1,2, \ldots, n, x \in \mathbb{R}_{+}^{n}, x_{i}=0,
$$

where $F_{i}$ is the ith coordinate of $\mathbf{F}$.

Proof. Let $x(t)$ be the solution of system (1) with the initial condition $x(0) \succeq 0$. In order to prove that $x(t) \geq 0$ for all $t \geq 0$, it is sufficient to check that the vector $\dot{x}(t)$ does not point toward the outside of $\mathbb{R}_{+}^{n}$. This is equivalent to verifying that the components of the vector $\dot{x}(t)=$ $\mathbf{F}(t, x(t))+w(t)$ corresponding to the zero components of $x(t)$ are nonnegative, which can be derived from condition (2) immediately. The proof of Proposition 2 is complete.

Definition 3 (see [21]). A vector field $\mathbf{f}: \mathbb{R}^{n} \rightarrow \mathbb{R}^{n}$ is said to be homogeneous of degree $p>0$ if $\mathbf{f}(\lambda x)=\lambda^{p} \mathbf{f}(x)$ for all $x \in \mathbb{R}^{n}$ and all $\lambda>0$.
Definition 4 (see [23]). A continuous vector field $\mathbf{f}: \mathbb{R}^{n} \rightarrow$ $\mathbb{R}^{n}$, which is continuously differentiable on $\mathbb{R}^{n} /\{0\}$, is said to be cooperative if the Jacobian matrix $(\partial \mathbf{f} / \partial x)(a)$ is Metzler for all $a \in \mathbb{R}^{n} /\{0\}$.

Denote by $f_{i}$ the $i$ th coordinate of $\mathbf{f}$. It is well known that a cooperative vector field satisfies the following Kamke condition (see [22, Remark 1.1, pp. 33]).

Proposition 5. Let $\mathbf{f}$ be a cooperative vector field. For any two vectors $x, y \in \mathbb{R}^{n} /\{0\}$ satisfying $x \geq y$ and $x_{i}=y_{i}$, one has $f_{i}(x) \geq f_{i}(y)$.

\section{Main Results}

We first consider the following particular case of system (1), where

$$
\begin{aligned}
\mathbf{F}(t, x) & \equiv \mathbf{f}(x), \\
w(t) & \geq 0, \\
\|w(t)\|_{\infty} & \leq r,
\end{aligned}
$$

$$
t \geq 0
$$

where the vector field $\mathbf{f}: \mathbb{R}^{n} \rightarrow \mathbb{R}^{n}$ is cooperative and $r>0$ is a constant. By Propositions 2 and 5, we see that system (1) is positive. For this case, we have the following necessary and sufficient condition for the reachable set bounding of system (1).

Theorem 6. Assume that (3) holds, and the vector field $\mathbf{f}$ : $\mathbb{R}^{n} \rightarrow \mathbb{R}^{n}$ is cooperative and homogeneous of degree $p>1$. Then the following two statements are equivalent.

(i) For any initial condition $x(0) \in \mathbb{R}_{+}^{n}$ and any disturbance $w(t)$ satisfying (3), there exist nonnegative constants $\alpha, \beta$, and $\gamma$ such that the solution $x(t)$ of system (1) satisfies

$$
\|x(t)\|_{\infty} \leq \alpha+(\beta+\gamma t)^{-1 /(p-1)}, \quad t \geq 0,
$$

where $\alpha$ depends on $r, \alpha=0$ if $r=0, \beta$ is related to $r$ and $x(0)$, and $\gamma$ is independent of $r$ and $x(0)$.

(ii) There exists an $n$-dimensional vector $v>0$ such that $\mathbf{f}(v) \prec 0$.

In addition, if condition (ii) holds, we can choose $\alpha=\theta \rho$, $\beta=(K \rho)^{1-p}$, and $\gamma=(p-1) \eta \rho^{1-p}$, where $\rho=\max _{i=1,2, \ldots, n} v_{i}$ and $\theta, K$, and $\eta$ are defined as follows:

$$
\begin{aligned}
\theta & =\left(\frac{r}{-\max _{i=1,2, \ldots, n} f_{i}(v)}\right)^{1 / p}, \\
K & = \begin{cases}0, & \|x(0)\|_{\infty}^{v} \leq \theta, \\
\|x(0)\|_{\infty}^{v}-\theta, & \|x(0)\|_{\infty}^{v}>\theta,\end{cases} \\
\eta & =-\max _{i=1,2, \ldots, n} \frac{f_{i}(v)}{v_{i}} .
\end{aligned}
$$

Proof. (ii) $\Rightarrow$ (i) Since system (1) is positive, each solution $x(t)$ of system (1) with the initial condition $x(0) \in \mathbb{R}_{+}^{n}$ satisfies 
$x(t) \geq 0$ for all $t \geq 0$. For any $\delta>1$, we can conclude from the definition of $K$ that

$$
\frac{x_{i}(0)}{v_{i}} \leq \theta+K<\delta(\theta+K), \quad i=1,2, \ldots, n .
$$

Set

$$
\begin{array}{r}
z_{i}(t)=\frac{x_{i}(t)}{v_{i}}-\delta \theta-\left[(\delta K)^{1-p}+(p-1) \eta t\right]^{-1 /(p-1)}, \\
t \geq 0, \quad i=1,2, \ldots, n .
\end{array}
$$

From (6), we have $z_{i}(0)<0$. By the continuity of $z_{i}(t)$ at $t=$ 0 , there exists a real number $t_{1}>0$ such that $z_{i}(t)<0$ for $i=1,2, \ldots, n$ and $t \in\left[0, t_{1}\right)$. We now prove that $z_{i}(t)<0$ for $i=1,2, \ldots, n$ and $t \geq 0$. Otherwise, there exist a real number $t_{2}>t_{1}$ and an index $k \in\{1,2, \ldots, n\}$ such that $z_{i}(t)<0$ for $i=1,2, \ldots, n$ and $t \in\left[0, t_{2}\right)$, and $z_{k}\left(t_{2}\right)=0$. Therefore,

$$
\begin{aligned}
& \dot{z}_{k}\left(t_{2}\right) \geq 0, \\
& \frac{x_{i}\left(t_{2}\right)}{v_{i}} \leq \delta \theta+\left[(\delta K)^{1-p}+(p-1) \eta t_{2}\right]^{-1 /(p-1)}, \\
& \quad i=1,2, \ldots, n, \\
& \frac{x_{k}\left(t_{2}\right)}{v_{k}}=\delta \theta+\left[(\delta K)^{1-p}+(p-1) \eta t_{2}\right]^{-1 /(p-1)} .
\end{aligned}
$$

By Proposition 5 and the homogeneity of $\mathbf{f}$, we obtain

$$
\begin{aligned}
f_{k} & \left(x\left(t_{2}\right)\right) \\
\quad \leq & f_{k}\left(\left[\delta \theta+\left((\delta K)^{1-p}+(p-1) \eta t_{2}\right)^{-1 /(p-1)}\right] v\right) \\
& =\left[\delta \theta+\left((\delta K)^{1-p}+(p-1) \eta t_{2}\right)^{-1 /(p-1)}\right]^{p} f_{k}(v) .
\end{aligned}
$$

From (1) and the definition of $z_{i}(t)$, we have

$$
\begin{aligned}
\dot{z}_{k}\left(t_{2}\right)= & \frac{\dot{x}_{k}\left(t_{2}\right)}{v_{k}}+\eta\left[(\delta K)^{1-p}+(p-1) \eta t_{2}\right]^{-p /(p-1)} \\
= & \frac{f_{k}\left(x\left(t_{2}\right)\right)+w_{k}\left(t_{2}\right)}{v_{k}} \\
& +\eta\left[(\delta K)^{1-p}+(p-1) \eta t_{2}\right]^{-p /(p-1)}
\end{aligned}
$$

By using the basic inequality $(a+b)^{q} \geq a^{q}+b^{q}$ for $a, b \geq 0$ and $q>1$, we get from (10) and (11) that

$$
\begin{aligned}
& \dot{z}_{k}\left(t_{2}\right) \\
& \quad \leq \frac{1}{v_{k}}\left[\delta^{p} \theta^{p} f_{k}(v)+r\right] \\
& \quad+\left[(\delta K)^{1-p}+(p-1) \eta t_{2}\right]^{-p /(p-1)}\left[\frac{f_{k}(v)}{v_{k}}+\eta\right] .
\end{aligned}
$$

Since $\delta>1$, by the definitions of $\theta$ and $\eta$, we obtain

$$
\begin{gathered}
\delta^{p} \theta^{p} f_{k}(v)+r<\theta^{p} f_{k}(v)+r \leq 0, \\
\frac{f_{k}(v)}{v_{k}}+\eta \leq 0 .
\end{gathered}
$$

Combining this with (12), we have $\dot{z}_{k}\left(t_{2}\right)<0$, which contradicts (8). Consequently, $z_{i}(t)<0$ for $i=1,2, \ldots, n$ and $t \geq 0$. That is, for any $\delta>1$,

$$
\begin{aligned}
\frac{x_{i}(t)}{v_{i}}<\delta \theta+\left[(\delta K)^{1-p}+(p-1) \eta t\right]^{-1 /(p-1)}, & \\
t & \geq 0, i=1,2, \ldots, n .
\end{aligned}
$$

As $\delta$ tends to 1 from the right, we have

$$
\begin{aligned}
& \frac{x_{i}(t)}{v_{i}} \leq \theta+\left[K^{1-p}+(p-1) \eta t\right]^{-1 /(p-1)}, \\
& t \geq 0, i=1,2, \ldots, n,
\end{aligned}
$$

which implies (4) with $\alpha, \beta$, and $\gamma$ defined as in Theorem 6.

(i) $\Rightarrow$ (ii) Assume that condition (4) holds for any initial condition $x(0) \in \mathbb{R}_{+}^{n}$ and any $w$ satisfying (3). In particular, each solution of the system $\dot{x}(t)=\mathbf{f}(x(t))$ with the nonnegative initial condition satisfies

$$
\|x(t)\|_{\infty} \leq(\beta+\gamma t)^{-1 /(p-1)} .
$$

That is, system (1) without disturbances is globally asymptotically stable. Invoking [23, Proposition 4.1], we have that there exists a vector $v>0$ such that $\mathbf{f}(v) \prec 0$. This completes the proof of Theorem 6 .

Next, we study the reachable set bounding for system (1) without condition (3). Based on a straightforward computation, we can get

$$
\begin{aligned}
D_{+}\left|x_{i}(t)\right| \leq F_{i}(t, x(t)) \operatorname{sign}\left(x_{i}(t)\right)+\left|w_{i}(t)\right|, & \\
i & =1,2, \ldots, n, t \geq 0,
\end{aligned}
$$

where $D_{+}\left|x_{i}(t)\right|$ denotes the derivative of $\left|x_{i}(t)\right|$ from the right and $w(t) \in B F_{r}\left([0, \infty), \mathbb{R}^{n}\right)$. The right-hand side of the above inequality is interpreted as $\left|F_{i}(t, x(t))\right|$ when $x_{i}(t)=0$.

Similar to Definitions 3 and 4 , a vector field $\mathbf{f}(t, x)$ : $[0, \infty) \times \mathbb{R}^{n} \rightarrow \mathbb{R}^{n}$ is said to be cooperative, if it is continuously differentiable on $\mathbb{R}^{n} /\{0\}$ with respect to $x$, and the Jacobian matrix $(\partial \mathbf{f} / \partial x)(t, a)$ is Metzler for $a \in \mathbb{R}^{n} /\{0\}$ and $t \geq 0$. The vector field $\mathbf{f}$ is said to be homogeneous of degree $p>0$ if $\mathbf{f}(t, \lambda x)=\lambda^{p} \mathbf{f}(t, x)$ for $x \in \mathbb{R}^{n}, \lambda>0$, and $t \geq 0$. In the following, assume that the vector field $\mathbf{F}$ satisfies the following.

(iii) There exists a vector field $\mathbf{f}(t, x):[0, \infty) \times \mathbb{R}^{n} \rightarrow \mathbb{R}^{n}$ which is cooperative and homogeneous of degree $p>1$, such that

$$
F_{i}(t, x) \operatorname{sign}\left(x_{i}\right) \leq f_{i}(t,|x|),
$$

$$
i=1,2, \ldots, n, t \geq 0, x \in \mathbb{R}_{n},
$$


where $F_{i}(t, x) \operatorname{sign}\left(x_{i}\right)$ is also interpreted as $\left|F_{i}(t, x)\right|$ when $x_{i}=0$.

Since $\mathbf{f}$ is cooperative and homogeneous, we can get from Propositions 2 and 5 that the following system is positive:

$$
\dot{x}(t)=\mathbf{f}(t, x(t))+|w(t)|, \quad t \geq 0 .
$$

By using the comparison principle, we have that solution $x(t)$ of system (1) with the initial condition $x(0) \in \mathbb{R}^{n}$ satisfies

$$
|x(t)| \preceq \bar{x}(t), \quad t \geq 0,
$$

where $\bar{x}(t)$ is the solution of system (19) with the initial condition $\bar{x}(0)=|x(0)|$. Consequently, by using Theorem 6 , we have the following sufficient condition for reachable set estimation of system (1).

Theorem 7. Assume that condition (iii) holds. If there exists a vector $v>0$ such that

$$
\mathbf{f}(t, v) \prec 0, \quad t \geq 0
$$

then for any initial condition $x(0) \in \mathbb{R}^{n}$ and any disturbance $w(t)$, the solution $x(t)$ of system (1) satisfies

$$
\|x(t)\|_{\infty}^{v} \leq \widetilde{\theta}+\left(\widetilde{K}^{1-p}+(p-1) \int_{0}^{t} \widetilde{\eta}(s) d s\right)^{-1 /(p-1)},
$$

$$
t \geq 0 \text {, }
$$

where

$$
\begin{aligned}
& \tilde{\theta}=\left(\max _{i=1,2, \ldots, n} \max _{t \geq 0} \frac{\left|w_{i}(t)\right|}{-f_{i}(t, v)}\right)^{1 / p}<+\infty \\
& \widetilde{K}= \begin{cases}0, & \|x(0)\|_{\infty}^{v} \leq \widetilde{\theta}, \\
\|x(0)\|_{\infty}^{v}-\widetilde{\theta}, & \|x(0)\|_{\infty}^{v}>\widetilde{\theta},\end{cases} \\
& \tilde{\eta}(t)=-\max _{i=1,2, \ldots, n} \frac{f_{i}(t, v)}{v_{i}} .
\end{aligned}
$$

Proof. Based on the above analysis, it is sufficient to prove that (22) holds for each solution of system (19) with any nonnegative initial condition. Denote $x(t)$ by the solution of system (22) with $x(0) \in \mathbb{R}_{+}^{n}$. For any $\delta>1$, let

$$
\begin{aligned}
& z_{i}(t)= \frac{x_{i}(t)}{v_{i}}-\delta \widetilde{\theta} \\
&-\left[(\delta \widetilde{K})^{1-p}+(p-1) \int_{0}^{t} \widetilde{\eta}(s) d s\right]^{-1 /(p-1)}, \\
& \quad t \geq 0, i=1,2, \ldots, n .
\end{aligned}
$$

Since $z_{i}(0)<0, i=1,2, \ldots, n$, we show that $z_{i}(t)<0$ for $t \geq 0$. Otherwise, there exist a real number $t_{2}>0$ and an index $k \in\{1,2, \ldots, n\}$ such that $z_{i}(t)<0, i=1,2, \ldots, n$, $t \in\left[0, t_{2}\right)$, and $z_{k}\left(t_{2}\right)=0$. It implies that $\dot{z}_{k}\left(t_{2}\right) \geq 0$,

$$
\begin{aligned}
& \frac{x_{i}\left(t_{2}\right)}{v_{i}} \\
& \quad \leq \delta \tilde{\theta}+\left[(\delta \widetilde{K})^{1-p}+(p-1) \int_{0}^{t_{2}} \tilde{\eta}(s) d s\right]^{-1 /(p-1)}, \\
& i=1,2, \ldots, n, \\
& \frac{x_{k}\left(t_{2}\right)}{v_{k}} \\
& =\delta \widetilde{\theta}+\left[(\delta \widetilde{K})^{1-p}+(p-1) \int_{0}^{t_{2}} \tilde{\eta}(s) d s\right]^{-1 /(p-1)} .
\end{aligned}
$$

Note that $\mathbf{f}(t, x)$ is a cooperative and homogeneous vector field of degree $p$. Based on the analysis in Theorem 6, we can eventually get

$$
\begin{aligned}
\dot{z}_{k}\left(t_{2}\right) \leq \frac{1}{v_{k}}\left[\delta^{p} \widetilde{\theta}^{p} f_{k}\left(t_{2}, v\right)+r_{k}\left(t_{2}\right)\right] \\
+\left[(\delta \widetilde{K})^{1-p}+(p-1) \int_{0}^{t_{2}} \tilde{\eta}(s) d s\right]^{-p /(p-1)} \\
\cdot\left[\frac{f_{k}\left(t_{2}, v\right)}{v_{k}}+\widetilde{\eta}\left(t_{2}\right)\right] .
\end{aligned}
$$

By the definitions of $\widetilde{\theta}$ and $\tilde{\eta}$, we conclude that $\dot{z}_{k}\left(t_{2}\right)<0$, which is a contradiction. The remainder of the proof is similar to that of Theorem 6 . This completes the proof of Theorem 7.

Remark 8. Compared with Theorem 6, we do not require in Theorem 7 that the initial condition $x(0) \in \mathbb{R}_{+}^{n}$ and the disturbance input $w(t) \geq 0$ for $t \geq 0$. Moreover, the disturbance $w(t)$ may be unbounded.

Remark 9. If $\int_{0}^{\infty} \tilde{\eta}(s) d s=+\infty$, then Theorem 7 guarantees that all the solutions of system (1) converge asymptotically within an ellipsoid.

Remark 10. Assume that there exists a vector field $\mathbf{g}: \mathbb{R}^{n} \rightarrow$ $\mathbb{R}^{n}$ such that $\mathbf{f}(t, x) \preceq \mathbf{g}(x)$ for $t \geq 0$ and $x>0$. Suppose also that there exist positive constants $r_{i}, i=1,2, \ldots, n$, such that $\left|w_{i}(t)\right| \leq r_{i}$ for $t \geq 0$. Then we get $\widetilde{\theta}=\left(\max _{i=1,2, \ldots, n}\left(r_{i} /-\right.\right.$ $\left.\left.g_{i}(v)\right)\right)^{1 / p}$. It can be seen from Theorem 7 that the bound of the reachable set is determined by the bound of disturbances, the choice of $v$, and the value of $p$. When the bound of disturbances and the value of $p$ are given, an appropriate vector $v$ can be chosen to guarantee a minimal bound of the reachable set by solving some nonlinear optimization problem (for details, please see analysis in the following numerical example). 


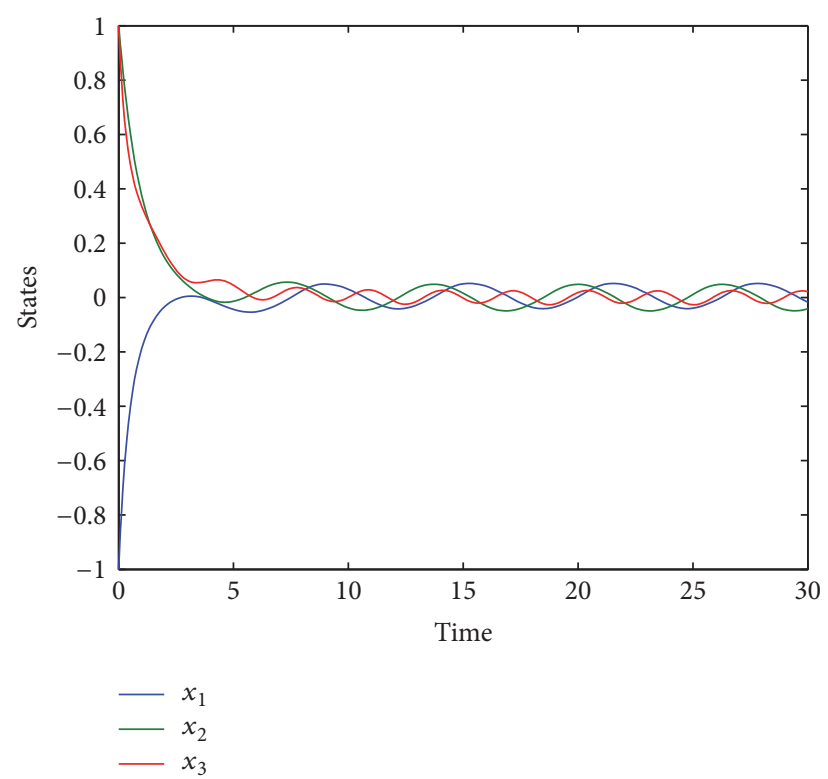

FIGURE 1: The state trajectories of system (1) with $x(0)=(-1,1,1)^{T}$.

\section{Numerical Example}

Consider system (1) on $\mathbb{R}^{3}$ with $w(t)=$ $0.05(\sin t, \cos t, \sin 2 t)^{T}$ and

$$
\mathbf{F}(t, x)=\left[\begin{array}{c}
-2\left|x_{1}\right|^{3 / 2} \operatorname{sign}\left(x_{1}\right)+\frac{t}{1+t} \sqrt{\left|x_{1} x_{2} x_{3}\right|} \\
(\sin t) \sqrt{\left|x_{1} x_{2} x_{3}\right|}-2\left|x_{2}\right|^{3 / 2} \operatorname{sign}\left(x_{2}\right) \\
(\cos t) \sqrt{\left|x_{1} x_{2} x_{3}\right|}-2\left|x_{3}\right|^{3 / 2} \operatorname{sign}\left(x_{3}\right)
\end{array}\right] .
$$

We see that condition (18) holds for the vector field

$$
\mathbf{f}(t, x) \equiv \mathbf{f}(x)=\left[\begin{array}{c}
-2\left|x_{1}\right|^{3 / 2}+\sqrt{\left|x_{1} x_{2} x_{3}\right|} \\
\sqrt{\left|x_{1} x_{2} x_{3}\right|}-2\left|x_{2}\right|^{3 / 2} \\
\sqrt{\left|x_{1} x_{2} x_{3}\right|}-2\left|x_{3}\right|^{3 / 2}
\end{array}\right]
$$

which is cooperative and homogeneous of degree $p=3 / 2$. If we choose $v=(1,1,1)^{T}$, then $(21)$ is valid. A straightforward computation yields that $\tilde{\theta}=0.05^{2 / 3}=0.1357$ and $\widetilde{\eta}(t) \equiv 1$. Therefore, all the solutions of system (1) converge asymptotically within the ball $\mathscr{B}(0.1357)$ by Theorem 7 . If we choose the initial condition $x(0)=(-1,1,1)^{T}$, then $\widetilde{K}^{1-p}=$ $(1-0.1357)^{-1 / 2}=1.0756$, and hence the solution $x(t)$ of the system satisfies $\|x(t)\|_{\infty} \leq 0.1357+(1.0756+0.5 t)^{-2}$. The simulation result is presented in Figure 1.

In the above numerical example, a better estimation of the reachable set can be obtained by choosing appropriate vector $v$. In fact, the minimal bound of the reachable set can be chosen to be $\min _{v>0} g(v)$ subject to $\mathbf{f}(v) \prec 0$, which is a nonlinear optimization problem, where $g(v)=(0.05 /-$ $\left.\max _{i=1,2, \ldots, n} f_{i}(v)\right)^{2 / 3} \max _{i=1,2, \ldots, n} v_{i}$.

\section{Conclusion}

In this paper, we have studied the problem of reachable set estimation for a class of continuous-time nonlinear time-varying systems with disturbances. When the involved system is a particular positive system, we establish a necessary and sufficient condition such that all the solutions of the system converge asymptotically within a ball. By using the comparison principle, an explicit sufficient condition for reachable set bounding of the general system is also presented. In both cases, the decay rate is estimated precisely. Finally, an illustrative example is given to demonstrate the effectiveness of the obtained result. The state bounding for both the continuous-time system with delay and the discretetime system is an interesting problem, which will be studied in the next paper.

\section{Conflicts of Interest}

The authors declare that they have no conflicts of interest.

\section{Acknowledgments}

This paper is supported by the National Natural Science Foundation of China under Grant nos. 61473133, 11671227, and 61374074 and the Natural Science Foundation of Shandong Province under Grant no. JQ201119.

\section{References}

[1] E. Fridman and U. Shaked, "On reachable sets for linear systems with delay and bounded peak inputs," Automatica. A Journal of IFAC, the International Federation of Automatic Control, vol. 39, no. 11, pp. 2005-2010, 2003.

[2] J.-H. Kim, "Improved ellipsoidal bound of reachable sets for time-delayed linear systems with disturbances," Automatica. A Journal of IFAC, the International Federation of Automatic Control, vol. 44, no. 11, pp. 2940-2943, 2008.

[3] Z. Zuo, D. W. Ho, and Y. Wang, "Reachable set bounding for delayed systems with polytopic uncertainties: the maximal Lyapunov-Krasovskii functional approach," Automatica. A Journal of IFAC, the International Federation of Automatic Control, vol. 46, no. 5, pp. 949-952, 2010.

[4] O. M. Kwon, S. M. Lee, and J. H. Park, "On the reachable set bounding of uncertain dynamic systems with time-varying delays and disturbances," Information Sciences. An International Journal, vol. 181, no. 17, pp. 3735-3748, 2011.

[5] P. T. Nam and P. N. Pathirana, "Further result on reachable set bounding for linear uncertain polytopic systems with interval time-varying delays," Automatica. A Journal of IFAC, the International Federation of Automatic Control, vol. 47, no. 8, pp. 18381841, 2011.

[6] P. T. Nam, P. N. Pathirana, and H. Trinh, "Exponential convergence of time-delay systems in the presence of bounded disturbances," Journal of Optimization Theory and Applications, vol. 157, no. 3, pp. 843-852, 2013.

[7] N. D. That, P. T. Nam, and Q. P. Ha, "Reachable set bounding for linear discrete-time systems with delays and bounded disturbances," Journal of Optimization Theory and Applications, vol. 157, no. 1, pp. 96-107, 2013. 
[8] Z. Zuo, Y. Chen, Y. Wang, D. W. Ho, M. Z. Chen, and H. $\mathrm{Li}$, "A note on reachable set bounding for delayed systems with polytopic uncertainties," Journal of the Franklin Institute. Engineering and Applied Mathematics, vol. 350, no. 7, pp. 18271835, 2013.

[9] J. Lam, B. Zhang, Y. Chen, and S. Xu, "Reachable set estimation for discrete-time linear systems with time delays," International Journal of Robust and Nonlinear Control, vol. 25, no. 2, pp. 269281, 2015.

[10] B. Zhang, J. Lam, and S. Xu, "Reachable set estimation and controller design for distributed delay systems with bounded disturbances," Journal of the Franklin Institute. Engineering and Applied Mathematics, vol. 351, no. 6, pp. 3068-3088, 2014.

[11] L. V. Hien and H. M. Trinh, "A new approach to state bounding for linear time-varying systems with delay and bounded disturbances," Automatica. A Journal of IFAC, the International Federation of Automatic Control, vol. 50, no. 6, pp. 1735-1738, 2014.

[12] P. T. Nam, P. N. Pathirana, and H. Trinh, "Reachable set bounding for nonlinear perturbed time-delay systems: the smallest bound," Applied Mathematics Letters. An International Journal of Rapid Publication, vol. 43, pp. 68-71, 2015.

[13] N. Zhang, Y. Sun, and P. Zhao, "State bounding for homogeneous positive systems of degree one with time-varying delay and exogenous input," Journal of the Franklin Institute, vol. 354, no. 7, pp. 2893-2904, 2017.

[14] L. Farina and S. Rinaldi, Positive Linear Systems: Theory and Applications, Wiley-Interscience, New York, NY, USA, 2000.

[15] T. Kaczorek, Positive $1 D$ and 2D Systems, Springer-Verlag, New York, NY, USA, 2002.

[16] X. Liu, W. Yu, and L. Wang, "Stability analysis of positive systems with bounded time-varying delays," IEEE Transactions on Circuits and Systems II, vol. 56, no. 7, pp. 600-604, 2009.

[17] X. Liu, W. Yu, and L. Wang, "Stability analysis for continuoustime positive systems with time-varying delays," Institute of Electrical and Electronics Engineers. Transactions on Automatic Control, vol. 55, no. 4, pp. 1024-1028, 2010.

[18] P. H. Ngoc, "On exponential stability of nonlinear differential systems with time-varying delay," Applied Mathematics Letters. An International Journal of Rapid Publication, vol. 25, no. 9, pp. 1208-1213, 2012.

[19] J. Qi and Y. Sun, "Global exponential stability of certain switched systems with time-varying delays," Applied Mathematics Letters. An International Journal of Rapid Publication, vol. 26, no. 7, pp. 760-765, 2013.

[20] H. R. Feyzmahdavian, T. Charalambous, and M. Johansson, "Exponential stability of homogeneous positive systems of degree one with time-varying delays," Institute of Electrical and Electronics Engineers. Transactions on Automatic Control, vol. 59, no. 6, pp. 1594-1599, 2014.

[21] J. G. Dong, "On the decay rates of homogeneous positive systems of any degree with time-varying delays," Institute of Electrical and Electronics Engineers. Transactions on Automatic Control, vol. 60, no. 11, pp. 2983-2988, 2015.

[22] J. K. Hale and S. M. Verduyn Lunel, Introduction to FunctionalDifferential Equations, Springer, Berlin, Germany, 1993.

[23] H. L. Smith, Monotone Dynamical Systems: an Introduction to the Theory of Competitive and Cooperative Systems, vol. 41 of Mathematical Surveys and Monographs, American Mathematical Society, Providence, RI, USA, 1995. 


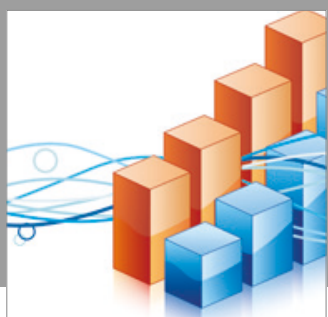

Advances in

Operations Research

vatersals

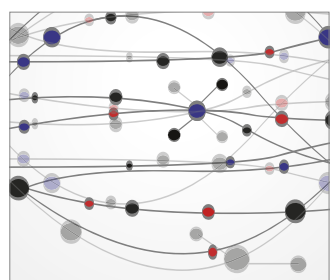

\section{The Scientific} World Journal
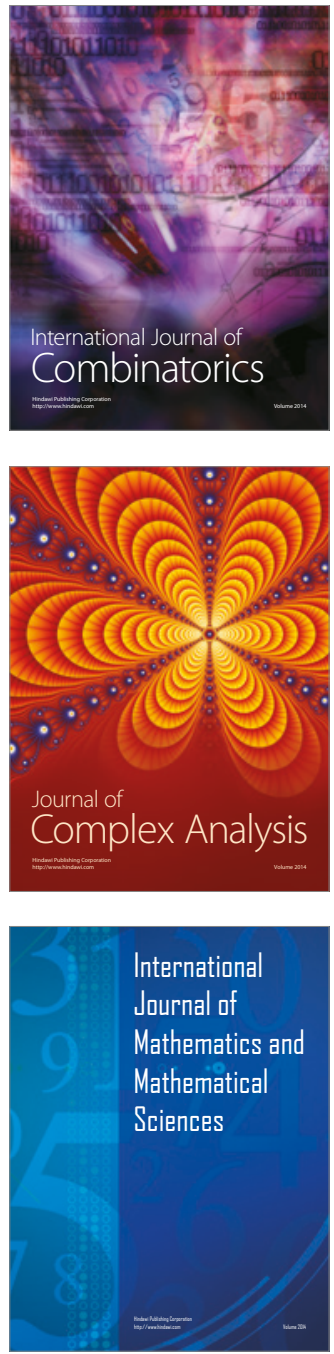
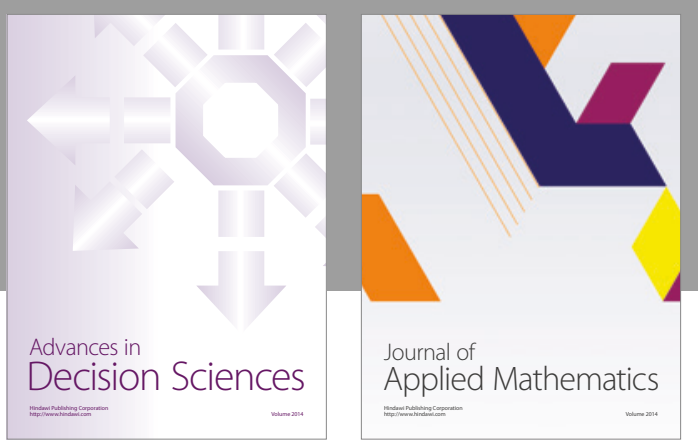

Algebra

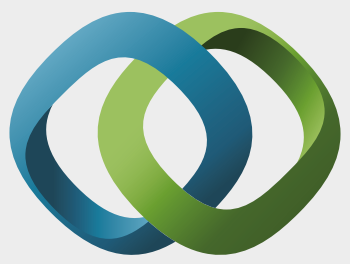

\section{Hindawi}

Submit your manuscripts at

https://www.hindawi.com
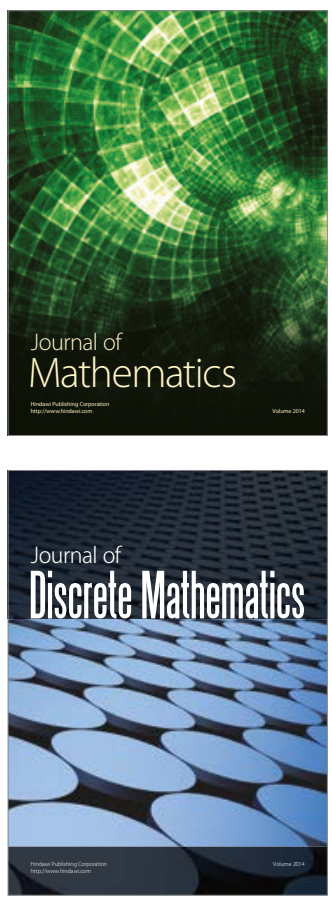

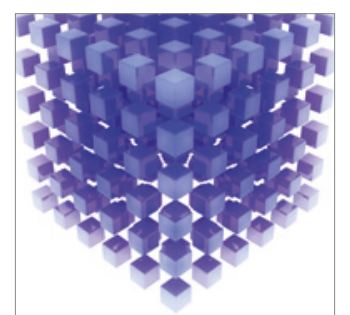

Mathematical Problems in Engineering
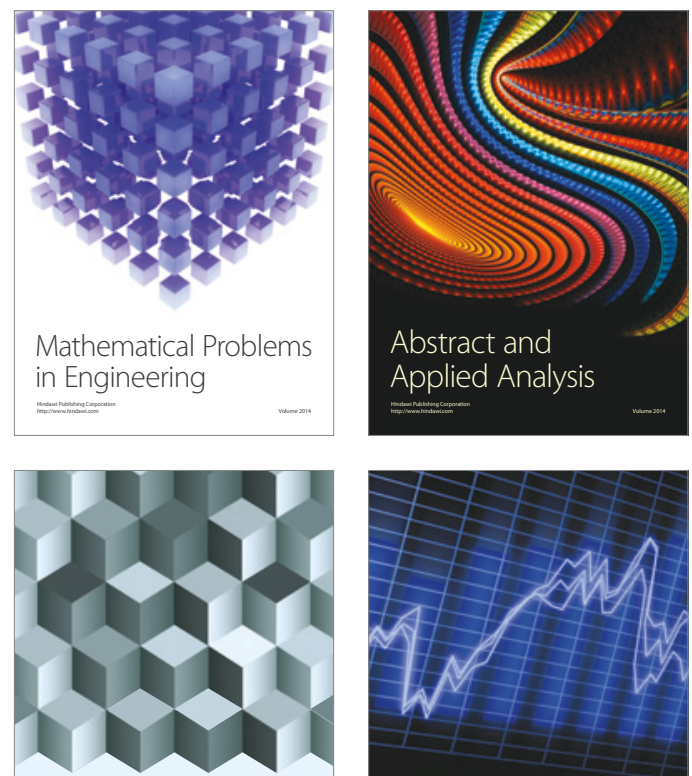

Journal of

Function Spaces

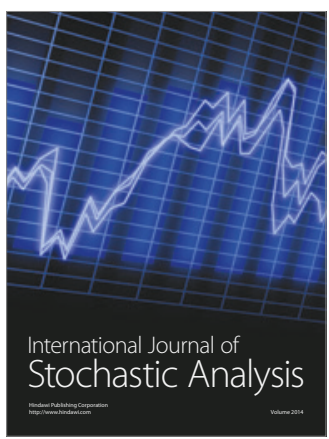

Probability and Statistics
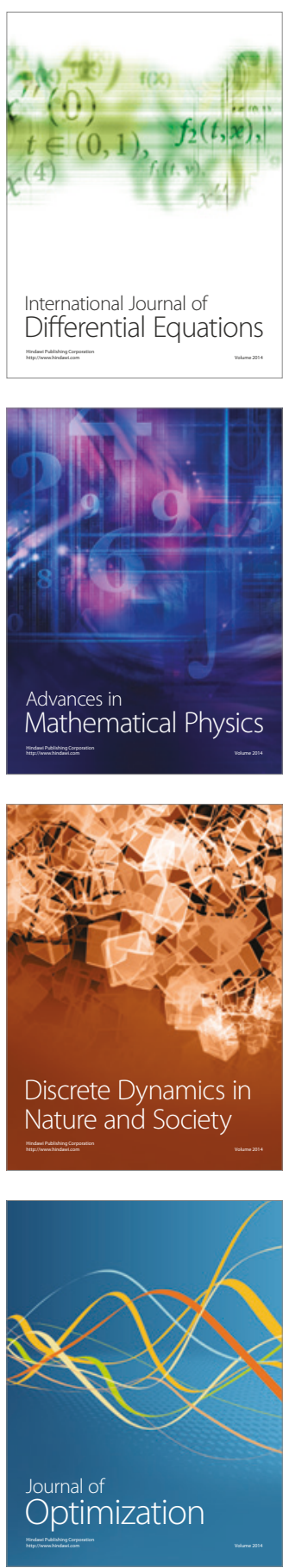